\title{
Study on Photoelectric Sensor to Detect the Concentration of $\mathrm{SO}_{4}{ }^{2-}$
}

\author{
FENG Ni ${ }^{1, a^{*}, \text { RUODAN Ni }}{ }^{2, b}$ and Ben Buryar ${ }^{2, c}$ \\ ${ }^{1}$ Department of materials science and engineering, Henan university of science and technology, \\ Luoyang 471003, China \\ ${ }^{2}$ Sannen laboratory of mechanics and dynamics, Luoyang 471023, China \\ anifeng@haust.edu.cn, bniruodan@163.com, 'Buryar@163.com
}

Keywords: electrolyte, concentration detection, photoelectric sensor, $\mathrm{SO}_{4}{ }^{2-}$

Abstract. We've designed a photoelectric sensor to detect the concentration of $\mathrm{SO}_{4}{ }^{2-}$ solution with a vertical capsule which was immersed into sample solution derectly. The system contains a pair of light-emitting diode (LED) and photosensitive diode (PIN) packaged with cover of semi-sphere shape. Theoretical analysis and experimental results have shown that the relative transmission rate of the system was almost proportional to the concentration of solution; which meant that the more concentrated the solution was, the less the relative transmission rate would be. In our experiments, the concentration of $\mathrm{SO}_{4}{ }^{2-}$ solution can be detected within the range of $0-3.441 \mathrm{~mol} / \mathrm{L}$ by using a LED with a central wavelength of $940 \mathrm{~nm}$. We have also confirmed that the concentration of $\mathrm{Zn}^{2+}(\leq 3.441 \mathrm{~mol} / \mathrm{L})$, $\mathrm{Cu}^{2+}(\leq 0.5627 \mathrm{~mol} / \mathrm{L})$ and $\mathrm{H}^{+}(\leq 2.015 \mathrm{~mol} / \mathrm{L})$ had tiny effects on the detection results of $\mathrm{SO}_{4}{ }^{2-}$ concentration.

\section{Introduction}

In the field of hydrometallurgy to obtain copper and zinc by means of electrolysis, $\mathrm{SO}_{4}{ }^{2-}$ ions always exist largely in the electrolytic solution. The degree and the change of its concentration rate always play an important role in determining the efficiency and the cost of the producing process, and also the quality of the products. For this reason, real-time detection and control of $\mathrm{SO}_{4}{ }^{2-}$ ion concentration are strongly required in practice. Nevertheless, chemical analysis, spectra analysis, polarographic analysis, chromatography analysis and some other means have been proved to be too complicated in operation, thus only suit for the offline detection. Recently with the rapid development of photoelectric technology, numerous of flexible, highly tight and highly compatible devices have appeared and come into use. They are often energy-efficient and have a small size, making it possible to develop a variety of innovational and portable online detection devices.

The method of absorption spectrophotometry based on the Lambert-Beer's law is often used to test the component of solution and it is favored for its sensibility, accuracy, simplicity and adaptability. It is almost possible to test all kinds of inorganic substances and a large number of organic ones directly or indirectly with this method. When using highly monochromatic light, the absorption spectrophotometry accords with the Lambert-Beer's law in a larger linear range, which means that it's capable to test a larger range of concentration rate. Semi-conductive LED can emit a range of light of different wavelengths, including the ultra-violet, visible and infrared light. With different LED and its corresponding photosensitive diode, an emitting-receiving system can be build up easily. Such system is simple, small size, energy-efficient and low-cost, therefore it draws people's attention to develop the absorption spectrophotometry method with semiconductor optoelectronic devices. [1]

Zhou Zuo-ping et al [2] developed an online concentration detection system by using a large-diameter glass waveguide and semiconductor optoelectronic devices. The system could be used to test the concentration of sugar solution and the limit was lower than $0.4 \%$. Du Wen-bin et al [3] designed an absorption spectrophotometry detection system based on microfluidic chip technology, they used an intensive and quasi-monochromic LED as the light source, a liquid-core waveguide as absorption cell and a photomultiplier as the receiver. A single-core silica fiber with a diameter of $500 \mu \mathrm{m}$ was set coaxially with the waveguide, coupling the waveguide and the photoelectronic devices. However, the liquid-core waveguide is high-cost and strongly requires very high stability and fine 
process accuracy of the system, which limit its portability and the use in online detection. Jing Qi et al [4] used an intensive green LED as the light source and a silicon photocell as the receiver to test the concentration of $\mathrm{Zn}^{2+}$ ions in solution. The sample solution filled up the glass tube with an inner diameter of $0.4 \mathrm{~mm}$; the LED and the silicon photocell were placed right to the port center of the glass tube. Ethanol was used as the reference solution to eliminate the effects of some unstable factors, like the fluctuation of light source, room temperature and the coupling condition of the system; dark room was used to eliminate the effect of the background light. This method requires that the light coming from the source should be entirely introduced into the sample solution by the port of the glass tube, while the light leaking into the wall of the glass tube will lower the accuracy of the detection.

Li Chun-yang et al [5] designed a micro photoelectric colorimetry system to detect the iron content of solution. The system was composed of five functional parts: embedded computer, micro photoelectric colorimetry device, auto titration apparatus, fluid adding-discharging system and stirring apparatus. The micro photoelectric colorimetry device adopted a double optical path of detection and reference, using an incandescent lamp as the light source and a silicon photocell as the light detector. It effectively avoids the disturbance caused by the change of characteristic parameters of photoelectric devices and the background light. However, this method is not practical in online detection since it uses thiocyanate to titrate the iron ions. Li Jian-guo et al [6] developed a method to test the chemical oxygen demand of water sample, they used an ultra-violet LED whose central wavelength is $255 \mathrm{~nm}$ as the light source and an ultra-violet photodiode as the receiver. Wang Chao et al [7] designed a compatible and portable pesticide residue detector by using a high-power monochromatic LED with a central wavelength of $410 \mathrm{~nm}$ as the light source and a silicon photocell with a central wave length of 550nm as the light detector. The light emitted by the LED penetrated through the solution sample in colorimetric cuvette, leading to a short circuit current of silicon photocell, which is proportional to the intensity of the transmitted light. Wang Feng-shuang et al [8] detected the concentration of different compositions in solutions by means of calculating the ratio of scattered light to transmitted light. They measured separately the intensity of scattered light and transmitted light at $90^{\circ}$ and $180^{\circ}$ relative to the incident direction and used the ratio to determine the concentration of casein solution. This method has the advantage to avoid measurement errors caused by the fluctuation of light power. In all these systems, the shapes and sizes of the colorimetric cuvettes strongly affect the detection results. By designing an absorption pool of solution sample with a reasonable size and shape, light absorption rate will be increased effectively and the accuracy of the detection can be improved [9]. However, the use of colorimetric cuvette makes the system to be inconvenient in real-time and online detection, which needs the sample solution to be charged and discharged.

Chen Shi-zhe et al [10] designed a system based on light position sensitive device (PSD) to detect the salinity of solution. The refractive index of solution changes accordingly to the concentration of dissolved salt, therefore changes the refraction angle of the incident light, causing a shift of the light received by PSD. The salinity of solution can be determined from the light shift tested by PSD. Such system can be used in online detection of the salinity of sea water, the resolution can reach $0.16667 \%$ wt. But the reliability and stability of the system are still in question due to the errors caused by multiple refraction and reflection of the light.

In conclusion, the structure of the capsule containing the solution sample and the relevant light way design are key points to detect the concentration of solution components by means of photoelectric sensing technology. They not only play an important role in improving the accuracy, sensitivity and stability of the detection result, but also directly determine the compatibility, simplicity, portability, maintainability and the real-time performance of the system. In this paper, we've designed a photoelectric sensor to detect the concentration of ion $\mathrm{SO}_{4}{ }^{2-}$ in solution, which had a vertical capsule immersed into test solution and a direct light way from LED to PIN. By immersing the capsule into sample solution, the concentration rate of ion $\mathrm{SO}_{4}{ }^{2-}$ can be obtained in real-time. 


\section{Composition of the system}

The compose of the sensing system is showed in Fig. 1. A vertical capsule with a filter at its bottom can be directly inserted into sample solution. A light-emitting diode (LED) and a photosensitive diode (PIN) packaged with transmitted cover of semi-sphere shape are oppositely embedded on the lateral wall of the capsule which is filled up by sample solution. The light coming out from the LED will go through the transmitted cover and the sample solution and enter into the receiving plane of the PIN on the other side. The LED is powered by a constant current supply and gives out the light with a certain intensity. The intensity of the light received by the PIN will change according to the concentration of the solution and a corresponding voltage output is given out from the PIN circuit. To eliminate the disturbance of background light, the wall of capsule is covered with a black coating.

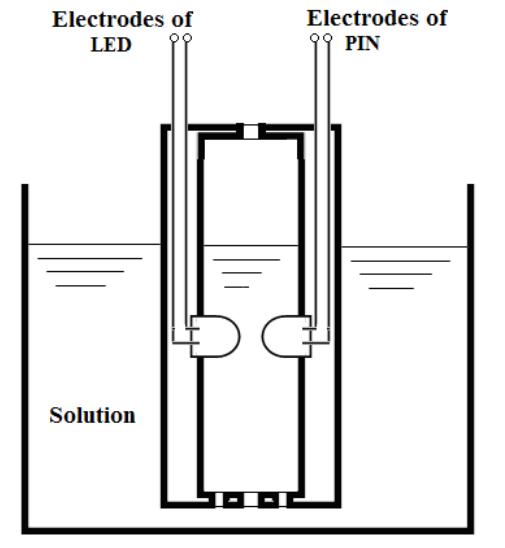

Fig. 1 Illustration of the photoelectric sensor

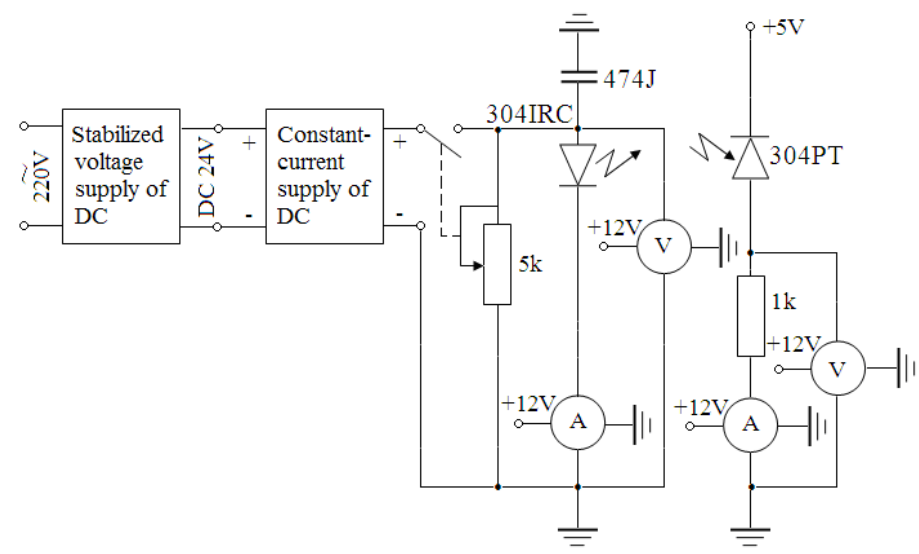

Fig. 2 Schematic diagram of the electric circuit

When using absorption spectrophotometry to test the composition of a sample solution, it is necessary to use a light source with wavelength corresponding to the peak absorption of the detected component to eliminate the influences of other components. Ion $\mathrm{SO}_{4}{ }^{2-}$ has two absorption peak: a strong one at the wavelength of $1210-1040 \mathrm{~nm}$ and a middle one at the wavelength of $680-570 \mathrm{~nm}$. In this paper we chose an infrared LED of type 304IRC as the experimental light source. Its central wavelength is $940 \mathrm{~nm}$, which is near to the range of the strong absorption peak of ion $\mathrm{SO}_{4}{ }^{2-}$. The photosensitive diode adopted is type 304PT. The circuit design of the system is showed in Fig. 2.

\section{Theoretical analysis}

The model of the light ways of the sensor is showed in Fig. 3. The LED light source is simplified as a point light source, while the receiving plane of PIN is simplified as a circular plane vertical to the axis with a radius of $a$. Such simplification would not alter the type of photoelectric transmit function of the system. As it is showed in Fig. 3, a light coming out from the LED, which has an angle $\theta$ with the center line of LED and PIN, will pass through the LED cover and the sample solution before it reaches the semi-spherical cover surface of the PIN. After being refracted by this cover, it arrives at the edge of circular receiving plane of PIN. The angle of incidence is $\beta$ and the angle of refraction is $\alpha$. We call $\theta$ as the critical rediation angle of the system. Suppose that the refractive index of the cover of PIN is $n_{\mathrm{o}}$ and that of the sample solution is $n_{\mathrm{s}}$. A group of formulas can be obtained as following according to the geometry relation in Fig. 3 and the refraction law of light.

$$
\begin{aligned}
& \beta=\gamma+\theta \\
& \frac{\sin \theta}{\sin \gamma}=\frac{\cos \theta}{L / R-\cos \gamma}
\end{aligned}
$$




$$
\begin{aligned}
& R \sin \gamma-a=b \cos \left(\alpha+\frac{\pi}{2}-\gamma\right)=b(\cos \alpha \sin \gamma-\sin \alpha \cos \gamma) \\
& b^{2}=a^{2}+R^{2}-2 a R \cos \left(\frac{\pi}{2}-\gamma\right)=a^{2}+R^{2}-2 a R \sin \gamma \\
& \frac{\sin \beta}{\sin \alpha}=\frac{n_{o}}{n_{s}}
\end{aligned}
$$

Where, $R$ is the radius of LED or PIN cover; $b$ is the distance from the critical light incidence point on the surface of PIN cover to the edge point on the receiving plane of PIN; $\gamma$ is the angle between the PIN cover radius passing through the critical light incidence point and the center line of LED and PIN; and $L$ is the distance between the center of LED and that of PIN. Combining Eq. 1 and Eq. 2, the following formula can be obtained.

$$
\sin \beta=\frac{L}{R \cos \theta(\operatorname{ctg} \theta+\operatorname{tg} \theta)}
$$

Combing Eq. 1, Eq. 3 and Eq. 4, the following formula can be obtained.

$$
\cos \alpha \sin (\beta-\theta)-\sin \alpha \cos (\beta-\theta)=\frac{R \sin (\beta-\theta)-a}{\sqrt{a^{2}+R^{2}-2 a R \sin (\beta-\theta)}}
$$

Combing Eq. 5, Eq. 6 and Eq. 7, the angle $\theta$ can be calculated by iteration method. Only a part of light emitting from the point source of LED, which within the range of cone angle $2 \theta$, can reach the receiving plane of PIN. The distance $\delta$ of the light passing through the sample solution is as following.

$$
\delta=R\left[\frac{L / R-\cos (\beta-\theta)}{\cos \theta}-1\right]
$$

Suppose that the intensity of light coming out from LED is $P_{L E D}$ and it is distributed uniformly on the surface area $2 \pi R^{2}$ of the semi-spherical cover. And also take the Lambert-Beer's law into account, the light intensity received by PIN from the LED is as following.

$$
P_{P I N}=P_{L E D} \int_{0}^{\theta_{C}} e^{-A \cdot C \cdot \delta} \sin \theta d \theta
$$

Where, $\theta_{C}$ is the critical rediation angle of the sample solution system; $\mathrm{C}$ is the $\mathrm{SO}_{4}{ }^{2-}$ concentration of the sample solution; $A$ is the optical absorption rate of ion $\mathrm{SO}_{4}{ }^{2-} ; \delta$ is a variable difined by Eq. 8 and Eq. 6. Suppose the working current of LED light source is $I$ and the output voltage of PIN circuit $V$, then we have relations as following.

$$
\begin{aligned}
& P_{L E D}=k_{L E D} \cdot I \\
& P_{P I N}=k_{P I N} \cdot V
\end{aligned}
$$


Where, $k_{L E D}$ is the electro-optic transfer rate of LED; $k_{P I N}$ is the reciprocal of PIN's photoelectric transfer rate. The transmission coefficient $k$ of the whole system is as following.

$$
k=\frac{V}{I}=\frac{k_{L E D}}{k_{P I N}} \cdot \frac{P_{P I N}}{P_{L E D}}=\frac{k_{L E D}}{k_{P I N}} \int_{0}^{\theta_{C}} e^{-A \cdot C \cdot \delta} \sin \theta d \theta
$$

The relative transmission rate $r$ of the sample solution to the reference solution is as following.

$$
r=\frac{k}{k_{0}}=\frac{\int_{0}^{\theta_{C}} e^{-A \cdot C \cdot \delta} \sin \theta d \theta}{\int_{0}^{\theta_{0}} e^{-A \cdot C_{0} \cdot \delta} \sin \theta d \theta}
$$

Where, $\theta_{0}$ is the critical rediation angle of the reference solution system; $C_{0}$ is the $\mathrm{SO}_{4}{ }^{2-}$ concentration of the reference solution; and $k_{0}$ is the transmission coefficient of reference solution system.

$$
k_{0}=\int_{0}^{\theta_{0}} e^{-A \cdot C_{0} \cdot \delta} \sin \theta d \theta
$$

When $C_{0}=0$,

$$
\begin{aligned}
& k_{0}=\int_{0}^{\theta_{0}} \sin \theta d \theta=1-\cos \theta_{0} ; \\
& r=\frac{1}{1-\cos \theta_{0}} \int_{0}^{\theta_{C}} e^{-A \cdot C \cdot \delta} \sin \theta d \theta .
\end{aligned}
$$

We should noticed that the effects of the reflection on the surface of PIN's semi-spherical cover and the absorption on the distance $b$ in PIN cover were neglected here. It was easly proved reasonable by calculations. In this case, the value of the incidence angle $\beta$ varies in the range of $31.6-33.8^{\circ}$, the reflectivity of the PIN cover surface should be lower than $5 \%$ and changed fractionally with solution concentration. The distance $b$ varies in the range of 0.9731-0.9834R and the PIN cover is transparent, so the absorption on the distance $b$ is very low and also changes tinily.

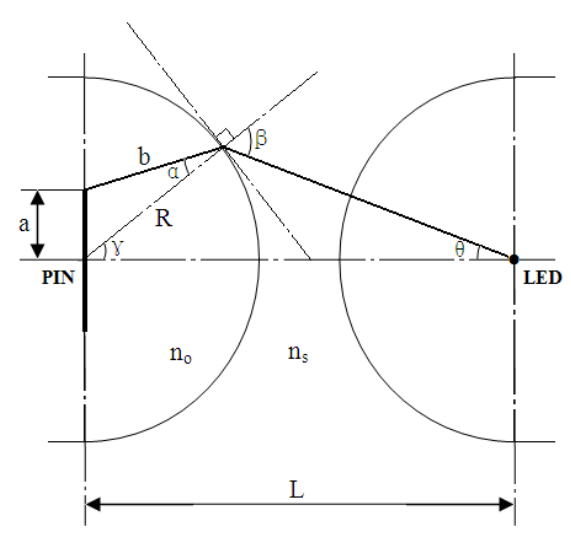

Fig. 3 Light way analysis of the sensor

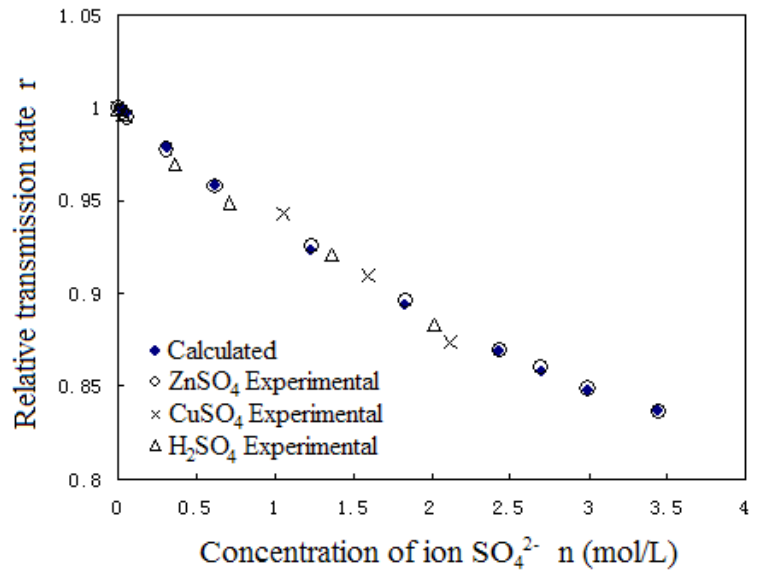

Fig. 4 Experimental and calculated results 


\section{Experimental and calculated results}

The experimental apparatus is set up according to the parameters listed in Table 1. We carried out both the experimental and theoretical analysis of the sample solutions of $\mathrm{ZnSO}_{4}, \mathrm{CuSO}_{4}$ and $\mathrm{H}_{2} \mathrm{SO}_{4}$. These solutions are prepared with deionized water and analytical pure $\mathrm{ZnSO}_{4} \cdot 7 \mathrm{H}_{2} \mathrm{O}, \mathrm{CuSO}_{4} \cdot 5 \mathrm{H}_{2} \mathrm{O}$ and $\mathrm{H}_{2} \mathrm{SO}_{4}$ respectively and the components are showed in Table 2 . The physical characteristics adopted in theoretical analysis is listed in Table 3. And the results are showed in Fig.4. It is obvious that within a large range of concentration, the relative transmission rate is nearly proportional to the concentration of $\mathrm{SO}_{4}{ }^{2-}(\leqslant 3.441 \mathrm{~mol} / \mathrm{L})$. The more concentrated the $\mathrm{SO}_{4}{ }^{2-}$ is, the less the relative transmission rate will be. Theoretical result has a good accordance with the experimental result. Furthermore, the concentration of $\mathrm{Zn}^{2+}(\leqslant 3.441 \mathrm{~mol} / \mathrm{L}), \mathrm{Cu}^{2+}(\leqslant 0.5627 \mathrm{~mol} / \mathrm{L})$ and $\mathrm{H}^{+}(\leqslant 2.015 \mathrm{~mol} / \mathrm{L})$ has tiny effect on the detection results of $\mathrm{SO}_{4}{ }^{2-}$ concentration.

Table 1 Parameters of photoelectric devices

\begin{tabular}{|c|c|c|c|c|c|}
\hline $\begin{array}{l}\text { Cover radius } \\
\mathrm{R}[\mathrm{mm}]\end{array}$ & $\begin{array}{l}\text { Center distance } \\
\mathrm{L}[\mathrm{mm}]\end{array}$ & $\begin{array}{l}\text { Refractive index } \\
\text { of PIN cover } n_{\mathrm{o}}\end{array}$ & $\begin{array}{l}\text { Peak wavelength } \\
\text { of LED } \lambda[\mathrm{nm}]\end{array}$ & $\begin{array}{l}\text { Half peak wavelength } \\
\text { of PIN } \lambda_{0.5}[\mathrm{~nm}]\end{array}$ & $\begin{array}{l}\text { Receiving plane } \\
\text { radius a [mm] }\end{array}$ \\
\hline 3.0 & 6.05 & 1.5159 & 940 & $700-1000$ & 1.5 \\
\hline
\end{tabular}

Table 2 Components of sample solutions

\begin{tabular}{|c|c|c|c|c|c|c|c|c|c|c|c|}
\hline Solution type & \multicolumn{10}{|c|}{ Concentration of ion $\mathrm{SO}_{4}^{2-}[\mathrm{mol} / \mathrm{L}]$} \\
\hline $\mathrm{ZnSO}_{4}$ & 0.006 & 0.031 & 0.062 & 0.312 & 0.618 & 1.234 & 1.846 & 2.429 & 2.700 & 2.995 & 3.441 \\
\hline $\mathrm{Cu}^{2+} / \mathrm{SO}_{4}{ }^{2-}=0.2658$ & 1.053 & 1.594 & 2.117 & & & & & & & & \\
\hline $\mathrm{H}_{2} \mathrm{SO}_{4}$ & 0.035 & 0.365 & 0.712 & 1.360 & 2.015 & & & & & & \\
\hline
\end{tabular}

Table 3 Solution physical characteristics adopted in theoretical analysis

\begin{tabular}{|c|c|c|c|c|c|c|c|c|c|c|c|c|}
\hline $\begin{array}{c}\text { Concentration of } \\
\mathrm{SO}_{4}^{2-}[\mathrm{mol} / \mathrm{L}]\end{array}$ & 0 & 0.006 & 0.031 & 0.062 & 0.312 & 0.618 & 1.234 & 1.836 & 2.428 & 2.700 & 2.995 & 3.441 \\
\hline Refractive index & 1.33475 & 1.3353 & 1.33543 & 1.3361 & 1.34315 & 1.3511 & 1.36646 & 1.3805 & 1.39245 & 1.39835 & 1.404 & 1.4091 \\
\hline $\begin{array}{c}\text { Absorption rate } \\
{[\mathrm{L} / \mathrm{mol} / \mathrm{mm}]}\end{array}$ & \multicolumn{10}{|c|}{24.436} \\
\hline
\end{tabular}

\section{Conclusions}

Theoretical and experimental results have shown that the concentration of solution could be detected by setting oppositely a pair of semi-sphere covered light-emitting diode (LED) and photosensitive diode (PIN) which were immersed into the sample solution. The concentration of certain component can change the light refractive index and absorption rate of the solution, thus change the transmission coefficient of the whole system. The relative transmission rate is nearly proportional to the concentration of the solution. The higher the concentration is, the less the relative transmission rate will be.

Using a LED with a central wavelength of $940 \mathrm{~nm}$ placed right in front of a PIN, the concentration of $\mathrm{SO}_{4}{ }^{2-}$ solution between them can be detected. Within the concentration range of $0-3.441 \mathrm{~mol} / \mathrm{L}$, the relative transmission rate is proportional to the concentration of $\mathrm{SO}_{4}{ }^{2-}$; the higher the concentration of $\mathrm{SO}_{4}{ }^{2-}$ is, the less the relative transmission rate will be. We have also confirmed that the concentration of $\mathrm{Zn}^{2+}(\leqslant 3.441 \mathrm{~mol} / \mathrm{L}), \mathrm{Cu}^{2+}(\leqslant 0.5627 \mathrm{~mol} / \mathrm{L})$ and $\mathrm{H}^{+}(\leqslant 2.015 \mathrm{~mol} / \mathrm{L})$ has tiny effects on the detection results of $\mathrm{SO}_{4}{ }^{2-}$ concentration.

\section{Acknowledgment}

Financial support came from No.0111040400, No.200410464002 and No.200510464022 of the natural science foundation of Henan province and No.2005ZD004 of the research foundation of Henan university of science and technology. 


\section{References}

[1] R.F.A. Wolffenbuttel, State of the Art in Integrated Optical Microspectrometers, IEEE Transactions on Instrumentation and Measurement, Vol.53(2004), No. 1: 197-202.

[2] Zuo-ping Zhou, Wei Lin, Investigation of Measurement of Solute Concentration in Line by Optoelectronic Method, Journal of Optoelectronics \& Laser, Vol. 2(1991), No.6: 338-339. (in Chinese)

[3] Wen-Bin Du, Qun Fang, Zhao-Lun Fang, A Microfluidic Chip for Absorbance Measurements with Long Optical Path-length Based on a Liquid-core Waveguide Technigue, Chemical Journal of Chinese Universities, Vol.25(2004), No.4: 610-613. (in Chinese)

[4] Qi Jing, Xueyi You, An Approach to Application of Micro-photoelectric Detection System to Environmental Monitoring, Shanghai Environmental Sciences, Vol.26(2007), No.5:189-192. (in Chinese)

[5] Chun-yang Li, Research on Embedded Measure Technology of Iron Concentration in Water, Master Thesis of Northeast Petroleum University, 2005. (in Chinese)

[6] Jian-guo Li, Research of UV Photoelectric Sensing System on Chemical Oxygen Demand of Water, Master Thesis of Yanshan University, 2013. (in Chinese)

[7] Chao Wang, Hui-zhong Zhu, Jian Wu, Wu-di Liang, Study of portable device for pesticide residue detecting with sampling rate emendation, Electronic Measurement Technology, Vol.30(2007), No.10:159-161, 171. (in Chinese)

[8] Feng-shuang Wang, Research on Detection Unit of Liquid Composition Concentration Based on Optical Scattering Method, Master Thesis of Harbin University of Science and Technology, 2014. (in Chinese)

[9] S. C. Terry, J. H. Jerman, J. B. Engell, A gas chromatographic air analyzer fabricated on a silicon wafer. IEEE Trans Electron Devices 1979, ED-26:1880 1886.

[10] Shi-zhe Chen, Nai-feng Cao, Yong Zhao, Kai-bo Hu, Research on seawater salinometer based on photoelectric sensor technology, Journal of Optoelectronics \& Laser, Vol. 20 (2009), No.12: 1622-2625. (in Chinese) 